\title{
Erratum to: Observed year-to-year sea surface salinity variability in the Bay of Bengal during the 2009-2014 period
}

\author{
Akurathi Venkata Sai Chaitanya - Fabien Durand - Simi Mathew • \\ Vissa Venkata Gopalakrishna • Fabrice Papa • Matthieu Lengaigne • \\ Jerome Vialard • Chanda Kranthikumar • R. Venkatesan
}

Published online: 8 February 2015

(C) Springer-Verlag Berlin Heidelberg 2015

\section{Erratum to: Ocean Dynamics}

DOI 10.1007/s10236-014-0802-x

The original version of this article unfortunately contained a mistake. Equations 2 and 3 were incorrect. The corrected equations are given below.

$$
\begin{aligned}
& \frac{d S^{\prime}}{d t}=\frac{1}{h A}\left[\begin{array}{c}
\iint \begin{array}{c}
\text { lateral } \\
\text { boundaries } \\
+\iint_{\text {ocean }} \\
\text { surface }
\end{array}\left[\left(E^{\prime}-P^{\prime}-R^{\prime}\right) \bar{S}+\overline{(E-P-R)} S^{\prime}\right] d s \\
\end{array}\right] \\
& \frac{d S^{\prime}}{d t}=\frac{1}{h A}\left[\iint_{\begin{array}{c}
\text { west and south } \\
\text { boundaries }
\end{array}} u_{\text {nomal }}^{\prime} \overline{\left(S_{\text {boundary }}-S\right)} d s+\iint_{\begin{array}{c}
\text { ocean } \\
\text { surface }
\end{array}}\left(E^{\prime}-P^{\prime}-R^{\prime}\right) S d s\right]
\end{aligned}
$$

The online version of the original article can be found at http://dx. doi.org/10.1007/s10236-014-0802-X.
A. V. S. Chaitanya $\cdot$ F. Durand $(\bowtie) \cdot$ V. V. Gopalakrishna C. Kranthikumar
CSIR/National Institute of Oceanography (NIO), Goa, India e-mail: fabien.durand@ird.fr

F. Durand · F. Papa

IRD/Laboratoire d'études en Géophysique et Océanographie,

Spatiales (LEGOS), Toulouse, France

\section{F. Papa}

Indo-French Cell for Water Sciences, IISc-NIO-IITM-IRD Joint

International Laboratory, IISc, Bangalore, India

S. Mathew $\cdot$ R. Venkatesan

National Institute of Ocean Technology (NIOT), Chennai, India

M. Lengaigne $\cdot J$. Vialard

Sorbonne Universités, UPMC Univ Paris 06, UMR 7159, LOCEAN,

Paris 75005, France

\section{Lengaigne}

Indo-French Cell for Water Sciences, National Institute of

Oceanography, Dona Paula, India 